\title{
LAS DIVISIONES TERRITORIALES EN LA CIUDAD DE CORDOBA
}

\author{
Alfonso MULERO MENDIGORRI*
}

\section{INTRODUCCION}

La ciudad, cada vez en mayor medida, es el marco espacial sobre el que se desarrollan multitud de análisis de la más variada índole - económicos, demográficos, sociológicos, geográficos,...- por parte de los estudiosos de la realidad urbana, entendida ésta en toda su extensión. A la vez, y como consecuencia de lo anterior, estamos asistiendo a un debate básico ${ }^{1}$ en torno a las divisiones territoriales empleadas tradicionalmente como soporte de los acercamientos científicos a los núcleos urbanos. No obstante, el problema se plantea en una doble vertiente ya que no sólo los citados especialistas han echado en falta unidades de análisis sólidas, coherentes, operativas y compatibles en la medida de lo posible. También la Administración Local - y en mayor grado si se quiere - ha padecido y padece esta necesidad, optando por ignorarla en la mayoría de los casos, y pasando por alto el apoyo técnico necesario en la elaboración de unas divisiones territoriales que, con carácter oficial, han configurado la base de la ordenación en las ciudades españolas durante décadas.

* Departamento de Geografía. Universidad de Córdoba.

1. Ver en este sentido los trabajos de Martín García, A.: «Sevilla: Puzzle de Divisiones Territoriales». Rev. de Estudios Andaluces, n. ${ }^{\circ} 7,1986$, pp. 61-75; y «Divisiones territoriales básicas y primarias en la ciudad de Sevilla: condiciones para una ordenación funcional de su espacio urbano». Ciudad y Territorio $\mathrm{n} .^{\circ}$ 68, 1986, pp. 119-123. Asimismo, son interesantes en esta línea desde el trabajo pionero de Abascal Garayoa, A. («Una aportación al estudio geográfico de la ciudad: la delimitación del barrio». Geographica, Julio-Diciembre de 1954, pp. 68-74) hasta los más recientes de Carreras i Verdaguer, C. («El barrio. Aspectos conceptuales de un elemento básico en la organización del espacio urbano». VII Coloquio de Geografía, A.G.E., Pamplona, 29 de Septiembre a 2 de Octubre de 1981, Tomo II, pp. 333-336) y Gaviria, C. («El barrio en los estudios de Geografía Urbana. Una propuesta de delimitación». VII Coloquio de Geografía A.G.E., Pamplona, 29 de Septiembre a 2 de Octubre de 1981, no publicado). 
Ante esta situación los que se han visto necesitados de un soporte territorial en sus estudios urbanos han obrado de diferente forma. Una línea de actuación viene definida por aquellos que han optado por adaptarse a las divisiones vigentes y utilizadas por la Administración, sin cuestionar la validez de las mismas y aceptándolas por la simple razón de su origen oficial ${ }^{2}$. De otra parte están los que han empleado algún tipo de unidad oficial o pseudoficial, señalando sus deficiencias y justificando su uso ante la carencia de otra alternati$\mathrm{va}^{3}$. Por último, una minoría de autores se ha enfrentado al problema realizando una compartimentación más coherente del espacio urbano que las existentes hasta el momento, buscando con ello dar una mayor solidez a su análisis, a la vez que lograr unos resultados más rigurosos ${ }^{4}$.

Por nuestra parte hemos pretendido en primer lugar un acercamiento a las divisiones territoriales empleadas por la Administración Local cordobesa, senalando sus principales características que, a grandes rasgos, son coincidentes en buena parte de las ciudades españolas. De otro lado, ante la necesidad de conjugar en una célula territorial básica los rasgos propios de la comunidad con la eficacia administrativa, desarrollamos un acercamiento conceputal al

2. La mayoría de los estudios que emplean una división de la ciudad como soporte se encuadran en esta línea de actuación, por ello sólo citaremos algunos a modo de ejemplo: Casas Torres, J.M. y Bodega Fernández, M.I.: «Primera nota sobre la distribución espacial de la población de Madrid en 1970» Geographica. Homenaje a Manuel de Terán, Enero-Diciembre de 1974, pp. 213-233; Abellán García, A.: «Estructura por sexo y edad de los distritos de Madrid» Estudios Geográficos, n. ${ }^{\circ} 144,1976$, pp. 303-317; Ocaña Ocaña, M.C.: Atlas Social de la Ciudad de Málaga. Málaga, Serv. de Publ. de la Universidad, 1984, 313 pp.; Jiménez Blasco, Beatriz C.: «La diferenciación sociodemográfica en los distritos municipales de Madrid» Aportación española al XXV Congreso Geográfico Internacional, París-Alpes, 1984, pp. 175-192; Del Pozo Grijalba, M.: «La estructura interna del municipio de Logroño: Análisis por distritos administrativos» Geographicalia, n. ${ }^{\circ}$ 5, 1980, pp. 71-111.

3. La argumentación de Cruz Villalón, J. y col, nos parece lo suficientemente ilustrativa de lo que ha sido esta otra forma de acercamiento al espacio urbano: «Ante la falta de una división administrativa válida y la inexistencia de cualquier otra consagrada por el uso, se utiliza en este artículo la delimitación zonal propuesta por GHESA-TEMA en Estudio para la planificación de las infraestructuras y servicios del transporte del área de Sevilla (PISTAS) (1983), en el que el municipio de Sevilla ha sido dividido en 130 zonas. No la consideramos, ni mucho menos, la división sectorial óptima de la ciudad, tanto más cuanto ha sido pensada con la finalidad específica de atender a la planificación del transporte urbano; pero tampoco vamos a entrar ahora en la crítica de dicha delimitación», ante esta situación concluyen que es necesaria la creación de «...unidades urbanas homogéneas, en las que, de acuerdo con la diferenciación sociológica creada, atender a necesidades comunes». En: Cruz Villalón, J. (Coor.): La población de Sevilla. Sevilla, Biblioteca de Temas Sevillanos, n. ${ }^{\circ} 33,1986$, pp. 18-19.

4. Ni que decir tiene que los trabajos encuadrados en esta línea son escasos. Sin embargo, estudios como el de Miralbes Bedera, R. y otros: «La variación espacial en la localización del comercio en La Coruña». Aportación española al XXV Congreso Internacional de Geografia. B.R.S.G., 1985; o como el de Calvo Palacios, J.L.: «Unidades de análisis y densidades urbanas: Zaragoza». Geographicalia, n. ${ }^{\circ} 5,1980$, pp. 5-32; han intentado solucionar la problemática de las unidades de análisis en el ámbito urbano. 
barrio con el convencimiento de que en la actualidad es la unidad con mayor arraigo en el conjunto urbano, y un soporte idóneo para la superación de esta antigua problemática territorial.

En último término proponemos unos criterios básicos de delimitación del barrio, que son síntesis de la base epistemológica señalada, la opinión de los propios habitantes de la ciudad y nuestra experiencia personal. El resultado de todo ello será la aplicación de esta metodología a la ciudad de Córdoba y, consecuentemente, la obtención de una nueva compartimentación del casco urbano cordobés, con el barrio como célula básica.

\section{CARACTERISTICAS DE LAS DIVISIONES TERRITORIALES DE ORIGEN ADMINISTRATIVO}

Desde muy antiguo los órganos administrativos locales vienen gestionando el espacio urbano valiéndose de una serie de divisiones territoriales a las que raramente se ha reconocido una utilidad fuera de su ámbito específico de aplicación. Las motivaciones que han impulsado su creación son de la más variada índole y, por ende, las divisiones que encontramos son diversas, heterogéneas y poco operativas una vez cumplido el objetivo para el que fueron creadas. Por otra parte, adolecen de un defecto fundamental como es su incompatibilidad, que les viene de no haber sido proyectadas, generalmente, con una perspectiva a largo plazo, ni con capacidad de adaptación a las posibles necesidades de análisis o estudios de la ciudad.

Nos centraremos a continuación en algunos ejemplos de divisiones administrtivas presentes en la ciudad de Córdoba, desentrañando sus principales características.

\subsection{Divisiones primarias}

La doble división en distritos y secciones se encuadra entre las calificadas como primarias porque, pese a sus modificaciones parciales, se ha configurado a lo largo del tiempo como el instrumento básico de ordenación interior y recogida de información en la mayor parte de las ciudades españolas.

En Córdoba los distritos se mantienen inalterables desde, al menos, $1955^{5}$, encontrándose el término municpal repartido entre una decena de ellos, cada uno de los cuales comprende un número desigual de secciones que suman en

5. González Portal, M.I, y Sanchez López, J.: «Evolución reciente de la población cordobesa (1960-1981)». Axerquia, n. ${ }^{\circ}$ 5, 1981, p. 19. 
conjunto 193. Actualmente esta división continúa siendo la utilizada por los órganos rectores del municipio en la administración del mismo y, por lo tanto, Censo de Población, Censo Electoral, Padrón Municipal de Habitantes, etc. se basan totalmente en ella. A grandes rasgos se caracteriza por:

1) De los diez distritos, cuatro son internos (3, 4, 7 y 8) y, como tales, imposibilitados para el crecimiento. Los seis restantes se abreu exteriormente hasta los límites del espacio municipal (Plano 1).

2) Utilizan como límites a las principales vías de comunicación y acceso a la ciudad, lo que resulta un criterio acertado, ya que las grandes arterias circulatorias desagregan el espacio urbano, otorgando a cada fragmento una singularidad que es normalmente sentida por los propios habitantes.

3) Coinciden los cuatro distritos internos casi totalmente con el casco histórico.

4) La variabilidad de su composición interna, puesto que las secciones que alcanzan los dos mil habitantes son divididas en el siguiente empadronamiento, aumentando su número progresivamente. Esto tiene el inconveniente de que al abordar, pongamos por caso, un estudio demográfico del municipio deberemos considerar previamente la estructura administrativa de cada momento censal, adaptándola a nuestra propias unidades de análisis.

5) La superficie de los distritos es excesivamente dispar, lo que a efectos de estudios comparativos puede resultar perjudicial. Tengamos en cuenta que mientras los cuatro distritos internos quedan reducidos prácticamente al caso histórico, los seis restantes se reparten desigualmente el resto de los $1.244,6 \mathrm{kms}^{2}$ con que cuenta el término municipal.

6) Cada distrito tiene un peso poblacional sustancialmente diferente, tanto en términos absolutos como relativos. Se trata de un rasgo común a buena parte de las ciudades españolas ${ }^{6}$, y el cuadro I muestra como este defecto nació prácticamente en los propios distritos.

6. Superficie y población son dos componentes que muy raras veces se encuentran equilibrados en los distritos de las ciudades españolas. Varios autores aportan datos que confirman este desfase en la planificación: Del Pozo Grijalba, M.: op. cit. pp. 71-111; Cruz Villalón, J. (coord.): Op. cit., p. 21; Abellán García, A.: op. cit., p. 304,... 
7) La mayoría de los distritos comprenden fragmentos urbanos heterogéneos, sea cual sea la óptica con que los contemplemos - económica, morfológica, cultural, etc.-. Esta es la característica que más desvirtúa el resultado de los estudios que adoptan la división en distritos como soporte territorial.

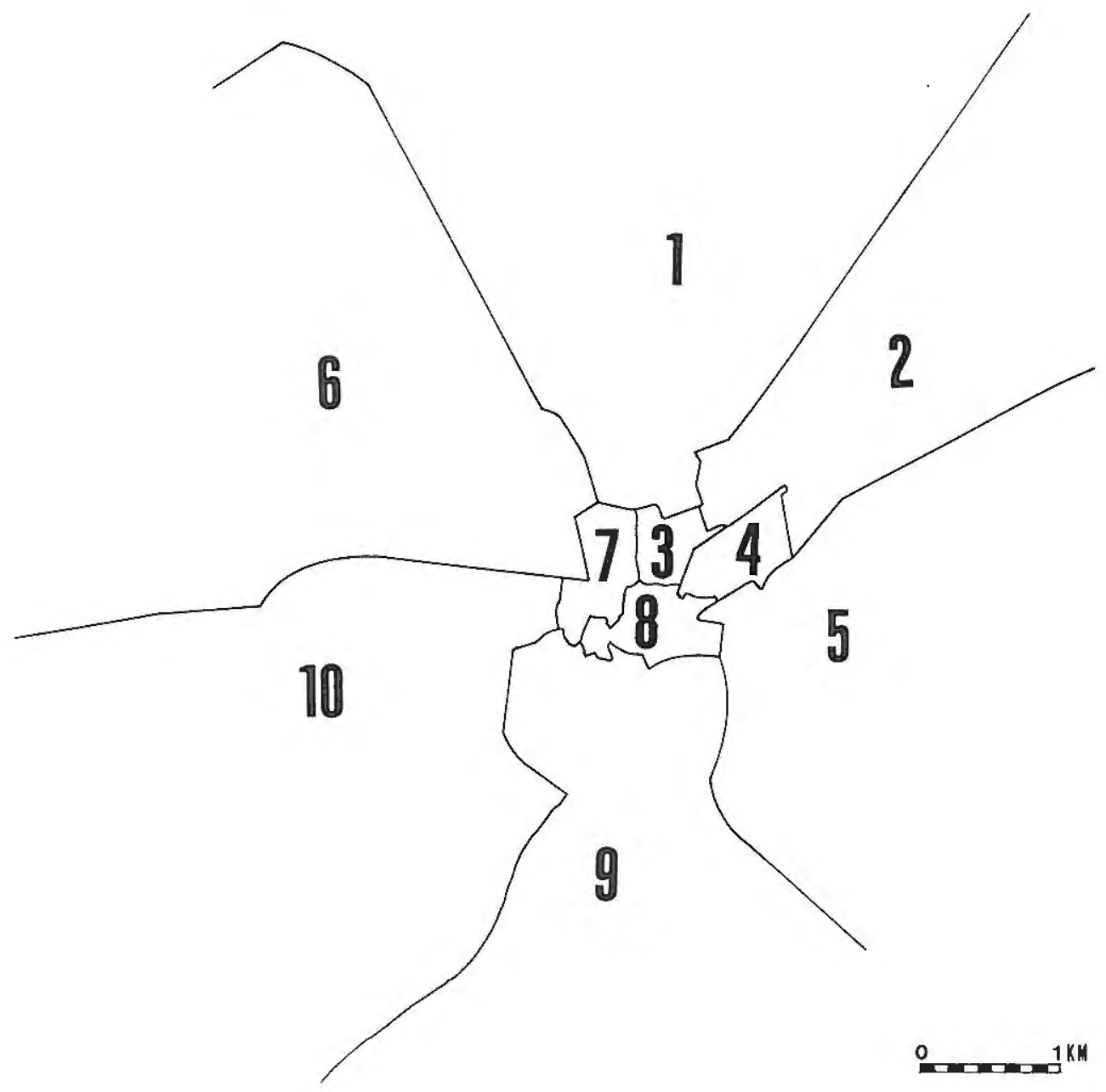

PLANO N. ${ }^{\circ} 1$

DIVISION EN DISTRITOS 


\section{CUADRO I}

DISTRIBUCION DE LA POBLACION CORDOBESA POR DISTRITOS (1955-1986)

\begin{tabular}{|c|c|c|c|c|}
\hline Distrito & 1955 & 1965 & 1975 & 1986 \\
\hline (................. & 23.150 & 24.556 & 28.655 & 39.696 \\
\hline 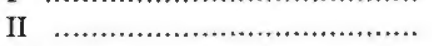 & 18.604 & 22.508 & 41.154 & 59.020 \\
\hline III & 6.892 & 5.527 & 3.018 & 3.494 \\
\hline IV & 10.710 & 8.048 & 5.180 & 4.993 \\
\hline V & 23.412 & 21.818 & 32.315 & 39.265 \\
\hline VI & 19.393 & 28.617 & 37.105 & 47.186 \\
\hline VII & 8.185 & 6.385 & 5.533 & 5.907 \\
\hline 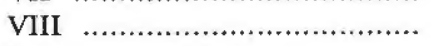 & 13.463 & 9.757 & 5.374 & 4.312 \\
\hline IX & 21.288 & 36.890 & 45.668 & 44.801 \\
\hline $\mathrm{X}$ & 37.120 & 51.681 & 46.007 & 46.616 \\
\hline
\end{tabular}

Fuente: Padrones Municipales de Habitantes. Elaboración propia.

El acercamiento a esta doble división confirma, por tanto, su inoperatividad como unidad de base para buena parte de los estudios de índole social. En Córdoba situaciones como la pertenencia de Electromecánicas y Ciudad Jardín - tan dispares socioeconómicamente- al mismo distrito $\left(10^{\circ}\right)$ o del Zumbacón y el Brillante al $\mathrm{n}^{\circ}{ }^{\circ}$, son suficiente motivo para desechar al distrito como unidad de análisis homogénea. Uno de los problemas radica en que las grandes dimensiones con que han sido diseñados facilitan que en su seno se localicen conjuntos con una morfología, una funcionalidad y unas características globales, en suma, radicalmente diferentes.

\subsection{Divisiones secundarias}

Paralelamente a los distritos y secciones, y a raiz de su ineficacia para adaptarse a otras necesidades operativas, se han venido elaborando desde el propio seno de la Administración —o en colaboración con ella- otras territorializaciones del municipio cordobés. La mayoría de ellas tienen un impacto espacial y vivencial mínimo, toda vez que han ido concebidas sin excesivo rigor. Pondremos dos ejemplos que consideramos ilustrativos por sus diferentes orígenes, desarrollos y objetivos.

De una parte encontramos los Consejos de Distrito, constituidos definitivamente el 15 de Noviembre de 1983 con la pretensión de superar la inoperancia de las Juntas Municipales de Distrito a las que sustituían. Se trata de una división territorial de Córdoba con las siguientes características: 
1) Su número se eleva ya a 15 , superando ampliamente a los distritos existentes en el municipio y sin conexión con ellos.

2) Su finalidad primaria es el fomento de la participación ciudadana ${ }^{7}$, organizada en base al marco territorial que estos Consejos ofrecen.

3) Su inoperancia como unidades básicas de gestión se manifiesta al considerar que la división en Consejos se ha diseñado a partir del territorio de acción de las Asociaciones de Vecinos, oganizaciones que están lejos de alcanzar una presencia homogénea en la ciudad. En la actualidad existen en Córdoba 55 Asociaciones de Vecinos desigualmente repartidas «ya que la mayoría se encuentran localizadas en barrios periféricos y marginales, mientras que en la zona centro no existe ninguna y en algunos barrios de alto nivel económico, como es Ciudad Jardín, han desaparecido» ${ }^{8}$.

Con una concepción completamente diferente aparecen las Areas del Plan General de Ordenación Urbana. Efectivamente este reciente Plan utiliza una división del municipio en 33 áreas como soporte de varios estudios parciales, sin que nos haya sido posible localizar en su texto justificación o explicación alguna de la citada división (¿por qué se denominan áreas?, ¿qué criterios se han seguido en su delimitación?, etc.). Un acercamiento pausado a tales áreas demuestra que varias responden a denominaciones que raramente se aplican en la realidad, pese a que en el estudio demográfico que dicho Plan contiene se afirma: «las áreas utilizadas como unidades de análisis espacial fueron confeccionadas por el equipo coordinador de los estudios previos (del PGOU) y responden a grandes rasgos con unidades de vecindad percibidas como tales por los propios habitantes de la ciudad» ${ }^{9}$.

Entre los principales defectos de esta nueva división territorial destacaremos los siguientes (ver Plano 2):

1) Ignora la existencia de barrios consolidados y nítidamente vividos en nuestra ciudad, tales como Fátima, Miraflores, Santuario y Valdeolleros.

7. En este sentido es conveniente señalar que cada Consejo de Distrito, además de una base territorial, comprende una organización interna compuesta por un presidente, no necesariamente concejal, un representante de cada una de las Asociaciones de Vecinos del Distrito, tres miembros elegidos directamente en asamblea y uno más por cada 25.000 ciudadanos.

8. Sarmiento Martín, E. y Sarmiento del Pueyo, M.E.: Consideraciones en torno a la participación ciudadana en el ámbito urbano. El caso de Córdoba. Córdoba, 1988, inédito, f. 14.

9. González Portal, M.I. y Sánchez López, A.J.: op. cit., p. 9. 


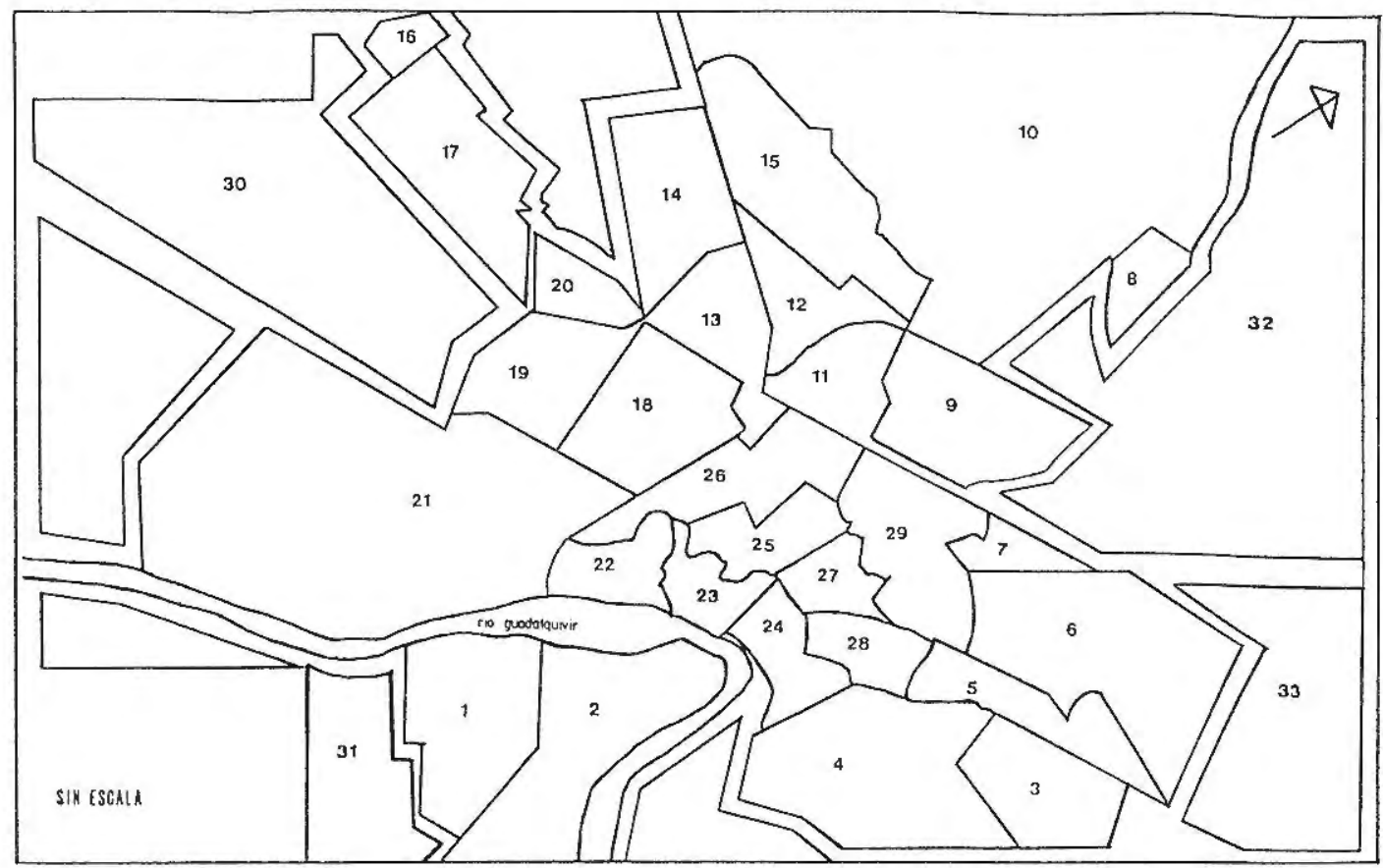

\section{PLANO N. 2}

AREAS (P.G.O.U.)

\section{LECTURA DEL PLANO}

1. Sector Sur

2. Campo de la Verdad

3. Cañero

4. Fuensanta

5. Levante Sur

6. Levante Norte

7. Zumbacón

8. Barrio Naranjo

9. Santa Rosa

10. Brillante

11. Huerta de la Reina
12. Moreras

13. Las Margaritas

14. Parque Figueroa

15. Albaida

16. Palmeras

17. Electromecánicas

18. Ciudad Jardín

19. Huerta de la Marquesa

20. Olivos Borrachos

21. Parque Cruz Conde

22. San Basilio
23. Mezquita

24. Axerquía

25. Tendillas

26. La Victoria

27. San Pablo

28. La Magdalena

29. Obispo Pérez Muñoz

30. Extrarradio Oeste

31. Extrarradio Sur

32. Extrarradio Norte

33. Extrarradio Este 
2) Los límites establecidos para ciertas áreas como Ciudad Jardín, Levante y Santa Rosa son excesivamente cuestionables ${ }^{10}$.

3) La división efectuada en el casco histórico —aunque no desconocemos la dificultad que esta operación entraña por la homogeneidad interna de gran parte de este fragmento urbano- además de imprecisa puede inducir a confusión puesto que otorga a ciertas áreas denominaciones erróneas, que se corresponden en realidad con zonas de menor dimensión real (el área que denominan Mezquita) o con otras de diferente emplazamiento histórico (la que denominan Ajarquía).

La conclusión, ante el panorama expuesto, es clara. La ciudad de Córdoba carece de una división territorial básica, operativa y flexible, y este hecho, por sí solo, contribuye a desvirtuar los presentes y futuros planes de ordenación territorial reduciendo, indudablemente, su efectividad. No obstante, tal problemática queda lejos de tener una localización aislada, ya que algunos de los escasos estudios en torno al tema confirman su presencia en diversas ciudades españolas $^{11}$.

\section{EL BARRIO COMO UNIDAD DE ANALISIS}

No cabe duda de que el barrio es la realidad más extendida cuando se habla de divisiones urbanas, siendo considerado a grandes rasgos como la unidad intermedia entre la calle y la ciudad. Tan generalizada concepción le otorga una ventaja sustancial frente a otras unidades urbanas de aparición más reciente y lo hace, por tanto, candidato a protagonizar una sólida división territorial de la ciudad. Esta división tendría como objetivo prioritario aunar dos polos frecuentemente distanciados: la realidad vivencial, socioeconómica y cultural

10. Hemos verificado - mediante encuesta directa a una muestra de los habitantes de la ciudadque algunas de las áreas propuestas por el P.G.O.U. son plenamente vividas y suficientemente diferenciadas en Córdoba; sin embargo, otras invalidan la citada división. Denominan Ciudad Jardín a un espacio mucho mayor que el barrio de dicho nombre, ya que incluyen también el sector comprendido entre Avda. Medina Azahara y Ronda de Cercadilla; se ignora la existencia de uno de los barrios más densamente poblados de la ciudad: Valdeolleros; el barrio de Fátima se identifica como Levante Norte, cuando es completamente individualizable tanto por sus dimensiones como por la percepción que de él se tiene. Asimismo aparece un grupo de áreas que responden a denominaciones muy puntuales - Tendillas, Jardines de La Victoria, Obispo Pérez Muñoz... - que difícilmente se identifican con el amplio espacio que se les ha asignado en la división. Ver: Avance del Plan General de Ordenación Urbana. Tomos I-1 y I-2, 1983.

11. Ver: Carreras i Verdaguer, C.: op. cit. p. 336; Martín García, A.: Divisiones Territoriales..., p. 126; Calvo Palacios, J.L.: op. cit. pp. 5 y ss. 
de los ciudadanos con las necesidades derivadas de la organización y eficacia administrativa. No obstante nos enfrentamos a un concepto intrincado que acertadamente Carles Carreras ha caracterizado como «evidente y oscuro a la vez» ${ }^{12}$. Este mismo autor, preocupado directamente por el debate en torno al barrio, repasó en su día las principales nociones de arquitectos-urbanistas, sociólogos y geógrafos por ser éstos los representantes de algunas de las disciplinas que con más profundidad han estudiado el barrio como unidad. Nosotros no vamos a dejarlas, obviamente, en el tintero dada la perentoria necesidad de clarificación conceptual. Sin embargo, perfilaremos ciertas definiciones, abundaremos en la concepción geográfica por ser la más completa con vistas a la ordenación del territorio, e introduciremos un ingrediente tan fundamental como es la concepción histórica.

\subsection{Concepción geográfica del barrio}

Pierre George, J.R. Johnston, Audrey N. Clark, Angel Abascal y José Luis Calvo Palacios son una muestra del interés del geógrafo en la definición y delimitacióu del barrio, y por ello es conveniente que atendamos a sus definiciones.

Pierre George afirma que «los barrios urbanos son a menudo antiguas unidades autónomas, que se diferencian en la evolución histórica de una ciudad por la composición de su población (...), o por su función antigua o actual (barrios de negocios, barrios comerciales, barrios industriales, barrios universitarios, etc.) o incluso por la edad de su construcción o de su inserción en la ciudad (barrios antiguos, barrios nuevos) o por su posición (barrios céntricos, barrios periféricos), (...). Sociológicamente el barrio es una entidad viviente en el interior de una ciudad y de esta forma es una realidad geográfica también; constituye un medio de vida, de actividades, de relaciones. Es percibido como un entorno inmediato, más familiar que el conjunto de la ciudad» ${ }^{13}$. El mismo George en otra de sus obras afirma: «El barrio es la unidad básica de la vida urbana, y posee un nombre que le confiere personalidad dentro de la ciudad» ${ }^{14}$.

Por su parte Johnston ofrece una definición con mayor matiz sociológico y con aportaciones novedosas: «(...) habitualmente dentro de un área urbana y en él dominan las relaciones cara a cara. Es una comunidad espacialmente delimitada, la existencia de la cual es a menudo más fácilmente percibida por

12. Carreras i Verdaguer, C.: op. cit., p. 333.

13. George, P.: Dictionnaire de la Géographie. P.U.F., París, 1970, p. 353.

14. George, P.: Geografía Urbana. Barcelona, Ariel, 1969 (2. ${ }^{\mathrm{a}}$ ed.), p. 94. 
los forasteros que por los residentes. Se asume que subculturas separadas existen en áreas particulares y que estas configuran las ideas y actividades de aquellos que viven allí (...)». Y concluye: «la delimitación geográfica precisa es difícil» ${ }^{15}$.

Tanto George como Johnston dejan claro el hecho de que el barrio tiene unos límites más o menos estables, que pueden quedar definidos atendiendo a diferentes criterios (históricos, de emplazamiento, de edad o funcionales según George, y en base a las relaciones humanas dominantes y a la percepción del individuo según Johnston), con lo que hemos dado un paso importante.

También es enriquecedora la definición de Audrey N. Clark: «Un barrio es un pequeño distrito habitado por gente en el que hay contactos sociales cercanos y cotidianos, y dentro del cual el indivíduo se siente seguro, en casa - la patria chica-. Los límites son indeterminados y más fácilmente discernibles por aquellos que vienen de fuera que por sus propios habitantes» ${ }^{16}$.

La evidencia de los contactos sociales y la mejor captación de los límites del barrio por las personas ajenas a él, son dos rasgos que manejan tanto Johnston como Clark, y que deben ser tenidos en cuenta en los proyectos de división de la ciudad en unidades de análisis.

Además de las anteriores aportaciones puramente teóricas, aunque no por ello menos válidas, otros geógrafos han intentado proporcionar una serie de pautas prácticas que lleven a una delimitación efectiva del barrio en el seno de la ciudad. En esta línea Angel Abascal, hace ya más de treinta años, constataba la imprecisión en el establecimiento de los límites del barrio, aportando tres criterios fundamentales de delimitación: funcional, morfológico y social; y alcaraba inmediatamente que, aunque distintos, son susceptibles de complementarse: «el criterio funcional se basa en el lugar ocupado por los habitantes del barrio, dentro de la ciudad. Su especialidad, laboral o no, les prestará una impronta muy particular: barrio comercial, industrial, administrativo, intelectual, militar, residencial, etc. El criterio morfológico utiliza datos más diversos: tipo y edad de las casas, materiales de construcción, disposición de las calles, forma del plano, etc. El criterio social tiene en cuenta el nivel de vida de los residentes habituales» ${ }^{17}$. Pese al interés del profesor Abascal por establecer un método riguroso y completo, no tiene en cuenta en ningún momento la opinión del vecino, del conocedor del barrio o del simple habitante de la ciudad.

15. Johnston, R.J. y otros: The Dictionary of Human Geography. Oxford, Basil Blackwell Publisher Limited, 1981.

16. Clark, Audrey N.: Longman Dictionary of Geography, Human and Physical. Harlow, Essex, Longman Group Limited, 1985.

17. Abascal Garayoa, A.: op. cit. p. 68. 


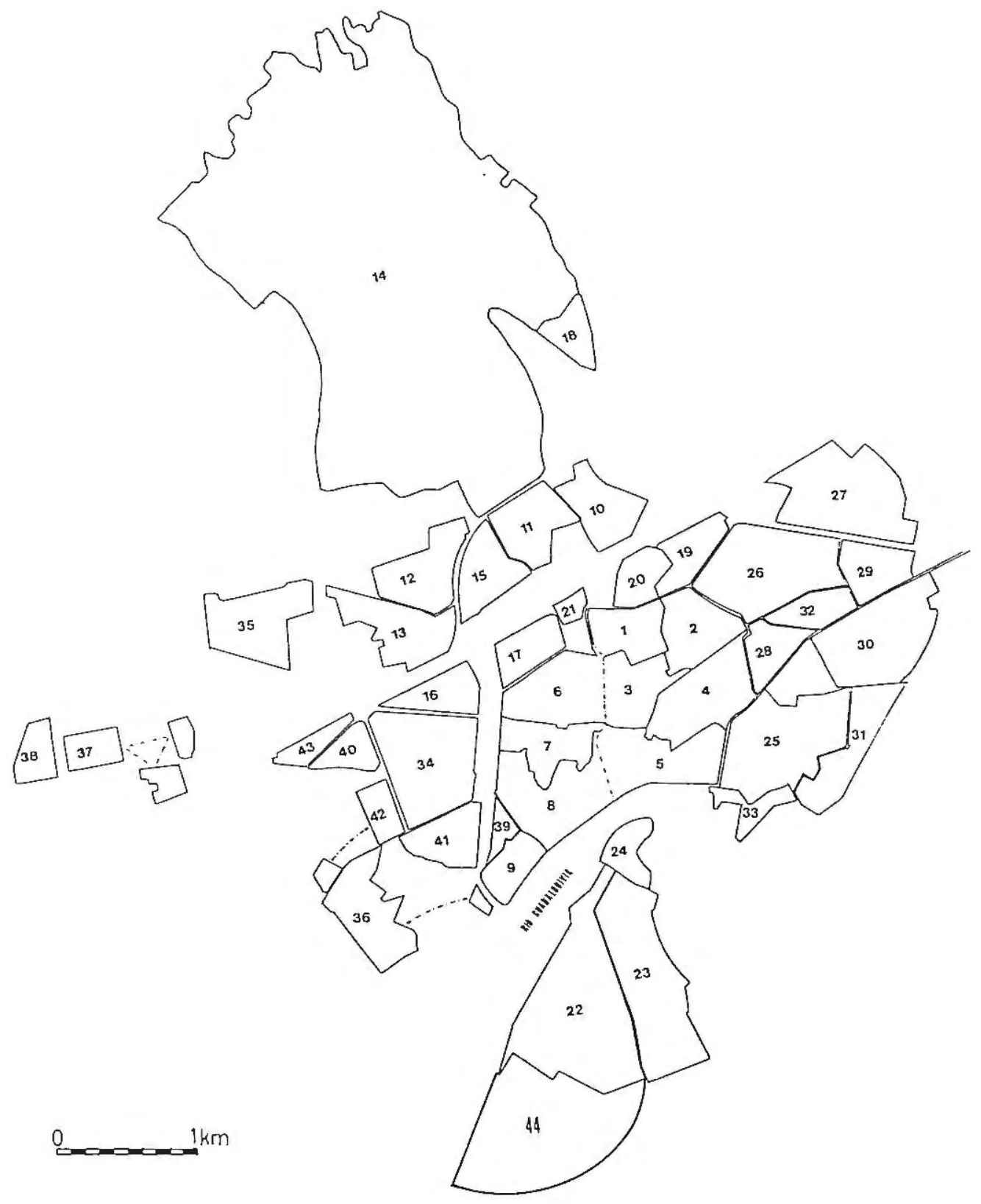

PLANO N. 3

DIVISION EN BARRIOS

[12] 


\section{LECTURA DEL PLANO}

\section{BARRIOS DE CORDOBA}

CASCO HISTORICO (Ajarquía)

1. Santa Marina

2. San Agustín

3. San Pablo

4. La Magdalena

5. San Pedro-La Ribera

CASCO HISTORICO (Villa)

6. Villa Alta

7. Villa Media

8. Villa Baja

9. San Basilio

\section{BARRIOS DEL NORTE}

10. Valdeolleros

11. Santa Rosa

12. Moreras

13. Margaritas

14. Brillante

15. Huerta de la Reina

16. Ronda de CercadillaMedina-Azahara

17. Ensanche

18. Barrio Naranjo

19. Zumbacón

20. Esperanza

21. Molinos Alta
BARRIOS DEL SUR

22. Sector Sur

23. Fray Albino

24. Miraflores

BARRIOS DEL ESTE

25. Fuensanta

26. Levante Norte

27. Fátima

28. Viñuela

29. Cairo

30. Cañero

31. Santuario

32. Benito de Baños

33. Arcángel

BARRIOS DEL OESTE

34. Ciudad Jardín

35. Parque Figueroa

36. Parque Cruz Conde

37. Electromecánicas

38. Palmeras

39. Vallellano

40. Huerta de la Marquesa

41. Vista Alegre

42. Polígono de Poniente

43. Olivos Borrachos

44. Polígono del Guadalquivir

En cada sector los barrios están numerados de mayor a menor, en orden a la población que acogen, excepto el «Polígono del Guadalquivir», incorporado a posteriori dada su reciente consolidación. 
En el mismo sentido que Abascal, J.L. Calvo Palacios aportó dos criterios de delimitación fundamentales: el morfológico y el funcional ${ }^{18}$.

\subsection{Concepción arquitectónico-urbanística}

En las visiones aportadas por los arquitectos urbanistas acerca del espacio interno de las ciudades ha predominado el componente morfológico. Según Carles Carreras, desde la consideración de la ciudad como un todo orgánico compuesto de células relativamente autónomas, y la paralela concepción del barrio como núcleo vital, se han desarrollado dos tendencias en la caracterización del barrio, poniendo ambas el peso de sus percepciones y delimitaciones en los aspectos morfológicos ${ }^{19}$. Una de ellas es de contenido más espiritual, y la sintetiza muy bien Kevin Lynch: «Un barrio urbano es, en su sentido más simple, un sector de carácter homogéneo, que se reconoce por claves que son continuas a través del barrio y discontínuas en otras partes. La homogeneidad puede ser de características espaciales (...), de tipo arquitectónico (...), de estilo o topografía. Puede ser una continuidad de textura, de color o de material, de superficie del piso, de escala o detalles de la fachada, de iluminación, arbolado o silueta. Cuanto más se superponen estos rasgos, más fuerte es la impresión de una región unificada» ${ }^{20}$. Y en otro párrafo afirma: «el observador entra en su seno (del barrio) mentalmente (...); siempre identificables desde el interior, también se los usa para la referencia exterior en caso de ser visibles desde fuera» ${ }^{21}$.

Sin embargo, entre las aportaciones de los arquitectos, los análisis de Aldo Rossi han sido fundamentales, y su concepción del barrio es considerada por algunos estudiosos del tema como la mejor, más crítica y operativa ${ }^{22}$ : «El barrio se convierte, por ello, en un momento, un sector de la forma de la ciudad, íntimamente vinculado a su evolución y a su naturaleza, constituido por partes, y a su imagen. De estas partes tenemos una experiencia concreta. Para la morfología social, el barrio es una unidad morfológica y estructural; está caracterizado por cierto paisaje urbano, cierto contenido social y una función propia; de donde un cambio en uno de estos elementos es suficiente para fijar el límite del barrio. También hay que tener en cuenta aquí el análisis del barrio como hecho social fundado en la segregación de clases o de razas, y en la función económica, o en todo caso en el rango social (...)» ${ }^{23}$.

18. Calvo Palacios, J.L.: op. cit., pp. 6 y ss.

19. Carreras i Verdaguer, C.: op. cit., p. 334.

20. Lynch, K.: La imagen de la ciudad. Barcelona, Gustavo Gili, 1984, p. 127.

21. Ibidem, p. 85 .

22. Carreras i Verdaguer, C.: op. cit. p. 335.

23. Rossi, A.: La arquitectura de la ciudad. Barcelona, Gustavo Gili, 1979, p. 105. 


\subsection{Concepción sociológica}

El componente sociológico en la percepción, definición y delimitación del barrio urbano está presente en los análisis de buena parte de las personas que han tratado este tema. Lo hemos detectado en las definiciones de casi todos los geógrafos, en las de Lynch y Rossi y ahora proponemos la que consideramos fundamental, enunciada por Suzanne Keller, que centrada específicamente en el terreno de la sociología se ocupa más del vecindario —entendido éste como el componente social del barrio- que del barrio como entidad global: «Dentro de sus fronteras físicas y simbólicas, un vecindario contiene habitantes que tienen algo en común, quizá sólo el medio ambiente que comparten. Esto le da un cierto carácter colectivo que influye y refleja los sentimientos de la gente sobre la vida en él y los tipos de relaciones que establecen los residentes» ${ }^{24}$. Y más adelante sostiene: «La existencia de vecindarios diferenciados en áreas urbanas ha sido estudiada mediante dos métodos principales, utilizando indicadores objetivos y subjetivos. En la utilización del método objetivo el investigador identifica y localiza áreas físicamente diferenciadas sobre la base de datos estadísticos y censuales, reconocimiento físico del terreno e información proporcionada por los informadores especialmente conocedores del área (...). Un enfoque subjetivo utiliza información sobre donde compra, trabaja y se divierte la gente de un área determinada, la distribución espacial de estas actividades proporciona la base para la fijación de límites (...). Otro enfoque pide a los mismos informadores que indique los límites y la extensión de sus vecindarios» ${ }^{25}$.

\subsection{Concepción histórica}

Las investigaciones de Torres Balbas contribuyen a aclarar el origen histórico del barrio, a la vez que despejan algunas confusiones. Así sucede con el frecuente error de, una vez establecido el origen musulmán del barrio, hacerlo derivar de la palabra arrabal ${ }^{26}$, cuando el término barrio aparecía claramente establecido en la semántica musulmana: «...las palabras arrabal —rabad-y barrio - hära- se empleaban con frecuencia indistintamente en la España musulmana, por lo que suelen confundirse ambas agrupaciones urbanas. El pri-

24. Keller, S.: El vecindario urbano. Una perspectiva sociologica. Madrid, Siglo XXI, 1975, p. 131.

25. Ibidem, pp. 135 y ss.

26. Carreras i Verdaguer, C.: op. cit., p. 333, afirma: «el gran ingeniero (refiriéndose a Ildefonso Cerdá) falló en la etimología al atribuir el origen del vocablo (barrio) a una deformación del burg germánico, ignorando su claro origen árabe que significa exterior, relativo a las afueras, arrabal». 
mer nombre se aplicaba casi siempre a una zona relativamente populosa, dentro de la cual había numerosos barrios de extensión variable, a veces formados tan sólo por una calle, pero no faltan ejemplos del caso contrario» ${ }^{27}$.

Por otra parte este autor insiste en que la desigual extensión de los barrios de las ciudades hispanomusulmanas ha llevado a que los diccionarios traduzcan la palabra con que se les designa en lengua árabe - hära y hawma en singular, härät en plural- unas veces por barrio y otras por calle ${ }^{28}$. En todo caso queda claro en la obra de Balbas que el barrio era una realidad presente en la configuración urbana de la época y en este sentido recoge los nombres de múltiples barrios perfectamente localizados en diferentes ciudades. Así, en la medina y arrabales de la Córdoba califal tenemos el barrio de los Zaŷyāli (funcionarios de la Corte), el hārat al-Tarrāzin (barrio de los tejedores o bordadores), el al-Raqqāqin (barrio de los pergamineros)... ${ }^{29}$. A la vista de estos y otros ejemplos puede pensarse que en su origen el barrio tendría una acepción de tipo gremial, si bien están perfectamente datados multitud de barrios donde no aparecía necesariamente una agrupación de gentes dedicadas al mismo menester. Otras veces barrios y arrabales venían definidos por su composición étnica, así las juderías, o barrios de los judíos, y las mozarabías o barrios de los mozárabes.

Por último, es interesante constatar que a partir de su origen islámico el barrio permaneció sólidamente integrado en las ciudades peninsulares y prueba de ello es que hacia 1500 se conservaban los nombres, castellanizados, de bastantes de los barrios de la Granada islámica, algunos de los cuales perduran en la toponimia urbana ${ }^{30}$.

En cualquier caso, de este acercamiento parcial a las principales nociones del barrio se desprende que la concepción geográfica es la más efectiva en la consecución de una delimitación del territorio urbano que pretenda apoyarse en aquel. La Geografía, como ciencia de síntesis, posibilita la confluencia de un mayor número de indicadores - funcionalidad, antigüedad, relaciones vivenciales, morfología, condición socioeconómica de los habitantes, etc.- necesarios todos ellos a la hora de alcanzar la división territorial integradora a que antes hacíamos alusión. No obstante, las visiones de la historia, urbanismo, sociología y otras disciplinas afines han de ser necesariamente tenidas en cuenta y sus principales aportaciones deben ser engarzadas con las específicamente geográficas.

27. Torres Balbas, L.: Ciudades Hispanomisulmanas. Madrid, Ministerio de Asuntos Exteriores, 1985, (2. ${ }^{\mathrm{a}}$ ed.), p. 179.

28. Ibidem, p. 190.

29. Ibidem, p. 191.

30. Ibidem, p. 192. 
Antes de seguir adelante, y a modo de recapitulación, creemos conveniente dejar sentadas las siguientes bases:

1) Que el barrio como tal es, entre otras cosas, una entidad geográfica diferenciada dentro de la ciudad y, por lo tanto, un marco adecuado para cualquier análisis que pretenda considerar las diferentes realidades espaciales.

2) Que los criterios para la delimitación del barrio pueden ser numerosos, pero no infinitos, y varios autores coinciden en la efectividad de su aplicación.

3) Que el barrio es una realidad viva y, como tal, mutable, por lo que «la delimitación solamente se justifica en aras de una simplificación artificial de la realidad que facilite el análisis, puesto que los hechos físicos o humanos no siempre tienen un punto y aparte que pueda apreciarse con nitidez $»^{31}$.

A continuación expondremos a grandes rasgos los criterios aplicados en la división de Córdoba en barrios.

\section{CRITERIOS BASICOS PARA LA DELIMITACION DEL BARRIO. LOS BARRIOS CORDOBESES}

Resulta evidente que una ciudad tan rica en matices como Córdoba no puede ser dividida atendiendo a un solo criterio, ya sea éste de base morfológica, funcional, perceptiva, etc. Por lo tanto, desde el primer momento nos propusimos adoptar una metodología mixta, que abarcara cuanta información fuese necesaria para conseguir una delimitación de los barrios coherente, sólida y vivida por los cordobeses en la medida de lo posible ${ }^{32}$. Conviene añadir que desde el comienzo estuvieron presentes cuatro situaciones que condicionaron la posterior delimitación, y que en cierto modo resumen el camino recorrido:

31. Calvo Palacios, J.L.: op. cit., p. 5.

32. No debe confundirse esta división en barrios con las existentes en otras ciudades españolas. Así tenemos la de Madrid de 1971 (Ver Bodega Fernández, M.I. y Casas Torres, J.M.: op. cit., p. 215) o la de Sevilla de 1971 (Ver Martín García, A.: «Divisiones...», p. 121), que no son sino una compartimentación de los distritos administrativos preexistentes en unidades menores a las que, infundadamente en muchos casos, se denomina barrios. Desde un punto de vista territorial estos supuestos barrios se encuentran a medio camino entre la superficie de la sección y del distrito, y respetando escrupulosamente los límites de este último aunque ello suponga fragmentar un barrio con identidad propia. Lamentablemente esta concepción del barrio como mera subdivisión del distrito administrativo esta muy extendída, ya que incluso es una de las acepciones más utilizadas por los diccionarios de la lengua (Ver, por ejemplo, el tratamiento que el Diccionario de la Real Academia de la Lengua 20 ed., da a este térnino). 
- La existencia de barrios que por una u otra característica $\longrightarrow$ por la conjunción de varias- están perfectamente diferenciados en la ciudad.

- La presencia de espacios plenamente vividos por sus habitantes, pero con unos límites difusos, e incluso sin una clara denominación.

- La complejidad de la división del casco histórico (Villa y Ajarquía) en barrios.

- La conveniencia de que los barrios respetaran —en la medida de lo posible y siempre que ello no supusiera la desfiguración de algún barrioel trazado de las secciones censales. La razón es doble: de una parte así será más fácil aprovechar a posteriori la información administrativa básica que nos ofrecen los Censos y Padrones; y en segundo lugar, con esta medida posibilitamos que la división resultante pueda ser empleada por los propios organismos de gestión municipal.

\subsection{Metodología}

A partir de estas premisas los pasos seguidos fueron los siguientes:

\subsubsection{Reconocimiento personal y detallado de la ciudad}

En base a este reconocimiento fuimos estableciendo, en unas tablas confeccionadas al efecto, las características que visualmente sirven para diferenciar cada parte de la ciudad. Se trataba de conocer lo siguiente: tipología edificativa, presencia de espacios intersticiales, obstáculos físicos destacados, funciones predominantes en cada zona, equipamientos públicos, densidad peatonal y de vehículos, características de los emplazamientos residenciales y características socioeconómicas básicas de los habitantes.

\subsubsection{Recopilación de información teórica}

Referida ésta a las diferentes partes de la ciudad, fundamentalmente en lo concerniente al período de implantación de cada una, el tipo de iniciativa que las hizo posibles y, en suma, a la evolución que han seguido hasta nuestros días. 


\subsubsection{Encuesta directa}

El reconocimiento visual y bibliográfico del territorio dejó lagunas importantes que procuramos solventar mediante la encuesta a vecinos y conocedores de cada parte de la ciudad. Fue de utilidad fundamentalmente en los siguientes casos:

- Cuando la denominación de una parte de la ciudad se desconocía o se prestaba a confusión.

- Cuando los límites entre barrios vecinos (con diferente denominación) eran confusos.

En lo referente a las personas sondeadas, no sólo fueron residentes en la zona, sino también foráneos conocedores de aquella, tipificados en tres personas: carteros, taxistas y pequeños comerciantes. Elección que en aquel momento consideramos acertada y que después vimos reforzada por las opiniones al respecto de Johnston y Clark ${ }^{33}$. Con anterioridad algunos autores (Lynch, por ejemplo) habían puesto en práctica el método de encuesta en este tipo de estudios urbanos obteniendo unos resultados muy positivos.

\subsection{Criterios utilizados en la delimitación}

Con la información de base conseguida tras el desarrollo de la citada metodología, más la aportación conceptual de los especialistas en el tema -antes expuesta sintéticamente- establecimos un conjunto de criterios complementarios que nos llevaron al trazado definitivo de los barrios cordobeses. A tales criterios objetivos unimos nuestra propio opinión en algunos casos puntuales.

\subsubsection{Criterio morfológico}

Ha sido revisado con detalle por Calvo Palacios ${ }^{34}$ que lo considera criterio fundamental en la delimitación de áreas de análisis. En nuestro caso hace alusión primordialmente, no exclusivamente, a la forma de las construcciones residenciales.

33. Johnston, R.J.: op. cit. p. 232; y Clark, A.N.: op. cit. p. 412, en su argumentación sobre la definición y delimitación de los barrios urbanos, coinciden en la cuestión de que los foráneos perciben mejor la realidad del barrio que, incluso, los propios residentes en él.

34. Calvo Palacios, J.L.: op. cit., p. 6. 
El barrio definido por este criterio contiene un caserío residencial homogéneo en buena medida, con una disposición de los ejes viarios sin grandes discordancias internas. Se trata de un criterio bastante extendido (compartido por el $75 \%$ de los barrios), lo que puede deberse en parte a lo tardío de la expansión cordobesa extramuros, cuyas iniciativas aisladas y su crecimiento «a saltos» han otorgado una morfología propia a cada barrio. Por su parte una gran porción del caserío del caso histórico mantiene una morfología añeja que Dominguez Ortiz ha descrito acertadamente ${ }^{35}$. Sin embargo, hay una serie de barrios $(25 \%)$ para los que sus características morfológicas no son definitorias de su individualidad o, en todo caso, ofrecen dudas. Sucede esto en Ciudad Jardín, donde está presente una amplia gama de edificación residencial —aún existen casas unifamiliares de dos plantas que contrastan con edificios de hasta nueve alturas-, lo cual queda compensado parcialmente por el trazado geométrico de sus vías principales y secundarias, que otorga unidad al conjunto. En otros casos como La Esperanza - donde coexisten la propia urbanización que le da nombre con toda la variedad edificativa de la calle Alonso El Sabio-, o como Las Margaritas — con una heterogenidad abrumadora fruto de su desordenada expansión- es prácticamente imposible una definición en base a este criterio morfológico.

\subsubsection{Criterio funcional}

Todos los barrios tienen una función predominante que los caracteriza, siendo ésta, en la mayor parte de los cordobeses, la residencial. Por este motivo nos hemos limitado a individualizar los barrios que, en lugar de la residencial o conjuntamente con ella, presentan otra función característica que ayude a definirlos. En este sentido, proponemos como ejemplos a la Villa Alta con una función comercial y de negocios; a la Villa Baja con un claro componente turístico; al Ensanche, con parecida función que la Villa Alta, pero con un marcado carácter residencial que no tiene aquella, etc. En el otro extremo destacan Las Moreras y Las Palmeras, cuyo carácter original de U.V.A.S. (Unidades Vecinales de Absorción) los define como barrios de alojamiento temporal para un determinado grupo social, más que propiamente residenciales. Creemos que en estos casos, más que en el resto, la función ha modelado la forma.

35. Dominguez Ortiz, A.: Andalucía ayer y hoy, Barcelona, Planeta, 1983, p. 50, dice así: «El núcleo antiguo de Córdoba, por ejemplo, a pesar de destrucciones parciales, se conserva en lo esencial, y aún destila ese aroma centenario, milenario casi, que hace de su caserío el más íntimamente andaluz. Aún se reconoce perfectamente el límite entre la Medina y la Ajarquía, aún es posible perderse en las callejas de la judería...». 


\subsubsection{Criterio vivencial}

Se trata de un criterio sumamente importante a la hora del estudio del barrio, tanto por lo valioso de la opinión de los propios habitantes como por el olvido a que tradicionalmente se ha visto sometido ${ }^{36}$. En la compartimentación que proponemos ha jugado un papel fundamental y creemos que es la plataforma sobre la que deberían apoyarse todos los intentos de ordenación territorial.

En el caso de Córdoba no ha ofrecido problema alguno en su aplicación y 30 de los 44 barrios de la ciudad aparecen como plenamente vividos por sus habitantes.

\subsubsection{Criterio de connotaciones socioeconómicas extremas}

Individualiza a ciertos barrios cuyo status socioeconómico hace que resulten diferenciados instantáneamente incluso por los foráneos. Los equipamientos del barrio, y el comportamiento de sus habitantes para con ellos; el aspecto exterior de las viviendas; el hacinamiento; la presencia o ausencia de luz y zonas ajardinadas; etc. creemos que son rasgos definitorios de un espacio residencial, y se hacen particularmente acusados cuando se comparan espacios ocupados por miembros pertenecientes a los dos extremos de la escala social. Tal es lo que sucede con la Villa Alta, Ensanche, Molinos, Alta, Brillante, Vallellano y Vista Alegre — todos ellos de alto standing- si los comparamos con el Zumbacón, Moreras y Palmeras, caracterizados por sus pésimas condiciones de habitabilidad. En conjunto son 12 los barrios que comparten este criterio, y es un indicador a tener muy en cuenta, pues está demostrado que «buena parte de los barrios mejor definidos son bastante atípicos en cierto modo por estar aislados o estar fuera del curso de la vida urbana, económicamente, como los barrios muy ricos o muy pobres; socialmente, como en los barrios pobres o en los suburbanos, y culturalmente, como en los guetos raciales o de inmigrantes» ${ }^{37}$.

36. En este sentido Carreras i Verdaguer, C.: op. cit., p. 336, insiste en la necesidad de considerar el punto de vista del habitante, en tanto que otros especialistas que han abordado expresamente la delimitación del barrio no han creido necesario tenerlo en cuenta (ver, por ejemplo, Abascal Garayoa, A.: op. cit., pp. 68 a 74).

37. Keller, S.: op. cit. p. 180. 


\subsubsection{Criterio histórico}

Aunque debe ser considerado en todas las ciudades, en Córdoba adquiere un protagonismo especial, ya que buena parte de ella está ocupada por un núcleo histórico que hasta el siglo XX fue prácticamente el único espacio residencial existente -excepción hecha de algunos arrabales extramuros_ 38 .

Este criterio por sí sólo caracteriza a los nueve barrios que se encuentran intramuros. En opinión de Suzanne Keller el criterio de identidad histórica es de los que más aportan en la definición de un vecindario (soporte vivencial del barrio): «Parece que la gente no identifica, por lo general, las subáreas donde vive por medio de nombres o de límites diferenciados, a no ser que tales áreas estén geográfica o socialmente aisladas o tengan una identidad histórica o de clase definida ${ }^{39}$.

\subsubsection{Criterio de emplazamiento excéntrico y/o aislamiento}

Se trata de un criterio que no es contemplado normalmente en estudios afines al que nos ocupa, puesto que suele ser considerado un ingrediente más del criterio morfológico.

En nuestro caso hemos creido conveniente tratarlo aparte a tenor de la especial configuración del municipio y de su ya señalada expansión a «saltos». Especial configuración porque Córdoba tiene en su seno dos verdaderos obstáculos que definen sus relaciones internas: el río Guadalquivir y la estación del ferrocarril. Expansión «a saltos» porque las iniciativas públicas y privadas anduvieron a la búsqueda a suelo barato, y éste lo era, en buena medida, en relación directa a su distancia al centro urbano - Cañero, Secem, Barrio Naranjo, Fray Albino,...-, salvo en los casos en que el traslado de la alta burguesía a la periferia revalorizó ese espacio -El Brillante-.

Diecinueve barrios bienen definidos en parte por este criterio. Los emplazamientos del Barrio Naranjo y de Las Palmeras son suficientemente ilustrativos de hasta donde puede llegar la desarticulación de uu municipio urbano de medianas proporciones (ver Plano 3).

38. López Ontiveros, A.: Evolución urbana de Córdoba y de los pueblos campiñeses, Córdoba, Diputación Provincial, 1981 (2. ${ }^{\mathrm{a}}$ ed.), pp. 135 y ss.

39. Keller, S.: op. cit., p. 145. 


\subsubsection{Criterio de límites físicos nítidamente establecidos}

Estos límites son, fundamentalmente, las principales arterias circulatorias -avenidas, ferrocarril,...-, el río Guadalquivir y los grandes espacios intersticiales y zonas verdes. Prácticamente los podemos identificar con lo que Lynch denominó «bordes» ${ }^{40}$, y son apreciables a simple vista o sobre un plano de la ciudad en los casos más claros del Barrio Naranjo y Olivos Borrachos —completamente aislados_; Ciudad Jardín y Ensanche - bordeados por cuatro avenidas fundamentales-; Sector Sur, Miraflores, Fray Albino y Polígono del Guadalquivir - separados del núcleo urbano por el río-, etc. Veinticinco barrios ostentan este criterio con claridad.

\subsubsection{Criterio de denominación inconfundible}

Corre paralelo al vivencial, pero se han dado situaciones en que los habitantes reconocen e individualizan una zona y, sin embargo, ya sea por su implantación reciente $u$ otro motivo, tal zona no responde a una denominación única e inconfundible. Lynch ${ }^{41}$ y Pierre George ${ }^{42}$ consideran a los nombres elementos importantes que hacen cristalizar la identidad de un barrio. Veintiocho barrios tienen en Córdoba un nombre sólidamente establecido, los mismos que comparten el criterio vivencial, salvo dos excepciones: Polígono de Poniente y La Esperanza, seguramente por su conformación reciente.

\subsubsection{Criterio étnico}

Este criterio es utilizado generalmente en los estudios anglosajones, pues en buena parte de sus ciudades de origen es frecuente la presencia de guetos de diferentes razas y nacionalidades. En nuestro caso nos hemos limitado a considerar el elemento gitano, puesto que contribuye a caracterizar ciertos barrios cordobeses, como Las Moreras, Las Palmeras o la zona alta del Sector Sur.

\footnotetext{
40. Lynch, K.: op. cit., p. 162.

41. Ibidem, p. 87.

42. George, P.: Geografía Urbana..., p. 94.
} 


\subsubsection{Reajustes del autor}

Por último, nuestro propio criterio ha debido ser aplicado a una serie de barrios detallados en el cuadro II. Parcialmente subjetiva ha sido la división del casco histórico ante el escollo que supone su gran homogeneidad interna en diversos sectores, y muy destacadamente en la Ajarquía. Subjetiva ha sido también la denominación otorgada a un grupo de barrios perfectamente individualizados, pero que carecían de un nombre único e inconfundible, lo que puede ser achacado en la mayoría de los casos a lo reciente de su implantación en la ciudad, que no ha permitido aún la gestación y difusión entre los habitantes del correspondiente nombre. Ronda de Cercadilla-Medina Azahara, Molinos Alta y Esperanza se encuentran en esta situación.

Los barrios de Benito de Baños, Cairo, Levante Norte y La Viñuela formaban parte del más amplio Polígono de Levante nombre bajo el que se les identificaba conjuntamente. No obstante, a tenor de su desmesurada superficie y del gran volumen de población que acogen creimos conveniente fragmentarlo en cuatro unidades que mantienen una personalidad propia dentro del extenso polígono. En estos casos las denominaciones empleadas, a excepción de Levante Norte, responden al nombre del eje viario principal de cada barrio.

Por último, el barrio del Brillante (amplia zona residencial de alto standing que se desarrolla por el piedemonte de la Sierra Morena cordobesa) ha sido delimitado al norte por la curva de nivel de $275 \mathrm{~m}$., que coincide a grandes rasgos con el final de la línea de máxima edificación.

Los 44 barrios resultantes tras el proceso descrito ${ }^{43}$ son, por lo tanto, aquellas partes de la ciudad en las que se deja sentir con fuerza uno de los criterios citados, o en las que confluyen varios de ellos. Siendo un barrio tanto más sólido cuanto más criterios comparta. El cuadro III refleja el reparto de la superficie y población urbanas en los barrios propuestos, así como el cuadro II muestra que en Córdoba los barrios mejor perfilados — seis o más criterios—son: Zumbacón, Moreras, Brillante, Sector Sur, Miraflores, Polígono

43. Llegados a este punto es conveniente aclarar que el reciente complejo residencial «Miralbaida» - emplazado en el sector Oeste de la ciudad — ha adquirido rápidamente una personalidad propia en el contexto de Córdoba y en el futuro, si los estudios previos así lo determinan, habrá de ser considerado un barrio más. También ha de quedar claro que los criterios propuestos por nosotros pueden y deben ser modificados o ampliados en cada caso si las circunstancias espacio-temporales lo requieren. Asimismo hay que incidir en que el grado de profundización en el estudio de cada criterio estará en función de las características de la ciudad y del propio barrio. Así, mientras que un barrio cualquiera quedará definido en función de, por ejemplo, la condición socioeconómica media de sus vecinos en base a una serie de datos elementales, otro requerirá un análisis profundo de esas mismas características socioeconómicas antes de establecer si queda o no individualizado por aquel criterio. 
CUADRO II

CRITERIOS DE DELIMITACION DE LOS BARRIOS CORDOBESES

\begin{tabular}{|c|c|c|c|c|c|c|c|c|c|c|c|}
\hline \multirow{2}{*}{ Barrios } & \multicolumn{11}{|c|}{ Criterios } \\
\hline & 1 & 2 & 3 & 4 & 5 & 6 & 7 & 8 & 9 & Total & Autor \\
\hline Santa Marina & * & & & & * & & & & & 2 & * \\
\hline 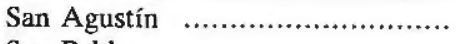 & * & & & & * & & & & & 2 & * \\
\hline San Pablo & * & & & & * & & & & & 2 & * \\
\hline La Magdalena & * & & & & * & & & & & 2 & * \\
\hline San Pedro-Ribera ...................... & * & & & & * & & & & & 2 & * \\
\hline Villa Alta & $*$ & * & & * & * & & & & & 4 & * \\
\hline Villa Media ............................. & * & & & & * & & & & & 2 & * \\
\hline Villa Baja & * & * & & & * & & & & & 3 & * \\
\hline 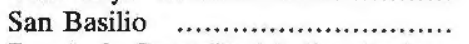 & $*$ & & * & & * & & * & * & & 5 & \\
\hline Ronda de Cercadilla-Medina Azahara & & & & & & & * & & & 1 & * \\
\hline Ensanche & * & * & & * & & & * & & & 4 & \\
\hline Molinos Alta & * & & & * & & & & & & 2 & * \\
\hline 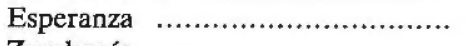 & & & * & & & * & & & & 2 & * \\
\hline Zumbacón $\quad$................................ & * & & * & * & & * & * & * & & 6 & \\
\hline 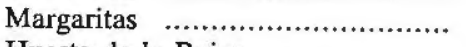 & & & * & & & * & & * & & 3 & \\
\hline Huerta de la Reina ................... & & & * & & & * & * & * & & 4 & \\
\hline 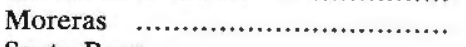 & $*$ & $*$ & * & * & & * & * & $*$ & * & 8 & \\
\hline 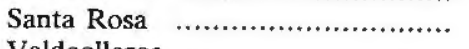 & * & & * & & & & * & * & & 4 & \\
\hline Valdeolleros & & & * & & & & * & * & & 3 & \\
\hline 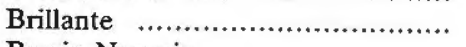 & * & * & $*$ & * & & * & * & * & & 7 & $*$ \\
\hline Barrio Naranjo . ....................... & * & & $*$ & & & * & * & * & & 5 & \\
\hline Sector Sur $\quad \ldots \ldots \ldots \ldots \ldots \ldots \ldots \ldots \ldots \ldots \ldots \ldots \ldots \ldots \ldots \ldots \ldots$ & $\star$ & & * & * & & * & * & * & * & 7 & \\
\hline
\end{tabular}


CUADRO II (Continuación)

\begin{tabular}{|c|c|c|c|c|c|c|c|c|c|c|c|}
\hline \multirow{2}{*}{ Barrios } & \multicolumn{11}{|c|}{ Criterios } \\
\hline & 1 & 2 & 3 & 4 & 5 & 6 & 7 & 8 & 9 & Total & Autor \\
\hline Fray Albino & * & & $*$ & & & * & * & * & & 5 & \\
\hline 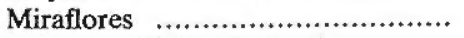 & $*$ & & $*$ & * & & * & * & * & & 6 & \\
\hline Polígono del Guadalquivir .......... & $*$ & & * & * & & * & * & * & & 6 & \\
\hline Viñuela $\quad \ldots \ldots \ldots \ldots \ldots \ldots \ldots \ldots \ldots \ldots \ldots \ldots \ldots \ldots \ldots \ldots \ldots \ldots$ & & & & & & & & & & 0 & $*$ \\
\hline Benito de Baños . ....................... & & & & & & & & & & 0 & $*$ \\
\hline Cairo & $*$ & & & & & & & & & 1 & $*$ \\
\hline Levante Norte . ........................ & & & & & & & & & & 0 & * \\
\hline 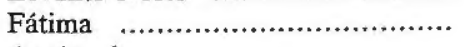 & $\star$ & & $*$ & & & $*$ & * & $*$ & & 5 & \\
\hline 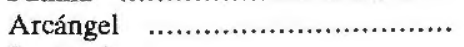 & & & $*$ & & & & & $*$ & & 2 & \\
\hline Santuario & * & & $*$ & & & & & * & & 3 & \\
\hline 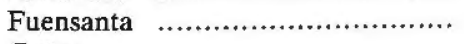 & * & & $*$ & $*$ & & & & * & & 4 & \\
\hline 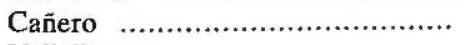 & * & & $*$ & & & * & * & * & & 5 & \\
\hline 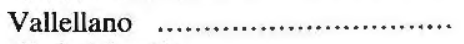 & $*$ & & * & * & & & & * & & 4 & \\
\hline Ciudad Jardín ......................... & $*$ & & $*$ & & & & $*$ & $*$ & & 4 & \\
\hline Huerta de la Marquesa .............. & $*$ & & $*$ & & & & * & $*$ & & 4 & \\
\hline Polígono de Poniente $. . . \ldots \ldots \ldots . . . .$. & * & & $*$ & & & & * & & & 3 & \\
\hline Vista Alegre & $*$ & & $*$ & * & & & * & * & & 5 & \\
\hline Parque Cruz Conde ................... & * & & $*$ & & & * & * & * & & 5 & \\
\hline Olivos Borrachos & $*$ & & $*$ & & & * & $*$ & * & & 5 & \\
\hline Electromecánicas $\quad$...................... & * & & * & & & $*$ & * & * & & 5 & \\
\hline 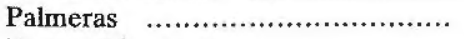 & * & $*$ & * & * & & * & * & * & * & 8 & \\
\hline Parque Figueroa & $*$ & & $*$ & & & * & $*$ & $*$ & & 5 & \\
\hline
\end{tabular}

Fuente: Elaboración propia. 
CUADRO III

DISTRIBUCION DE LA POBLACION Y SUPERFICIE DE LOS BARRIOS CORDOBESES (1984)

\begin{tabular}{|c|c|c|}
\hline Barrio & Población (Habs.) & Superficie (Has.) \\
\hline 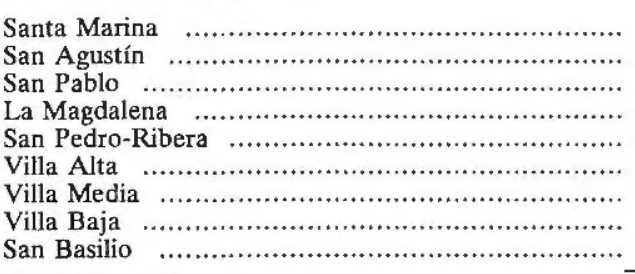 & $\begin{array}{l}4.219 \\
4.705 \\
3.483 \\
5.092 \\
5.716 \\
6.565 \\
4.091 \\
3.910 \\
1.194\end{array}$ & $\begin{array}{l}18,27 \\
22,12 \\
20,02 \\
31,70 \\
30,77 \\
31,62 \\
19,82 \\
35,52 \\
13,42\end{array}$ \\
\hline Total Casco Histórico $\quad$.......................................... & 38.975 & 223,29 \\
\hline 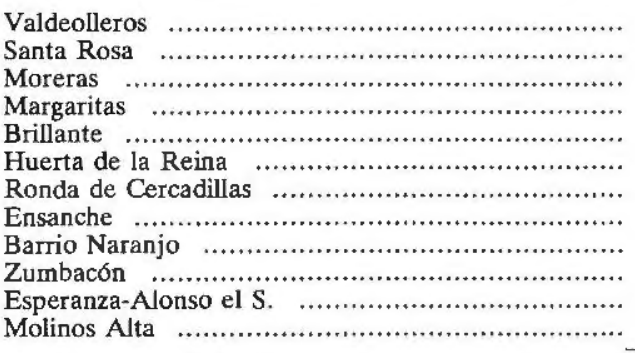 & $\begin{array}{r}11.028 \\
7.759 \\
7.389 \\
6.680 \\
6.253 \\
5.254 \\
4.091 \\
3.952 \\
3.851 \\
2.531 \\
2.015 \\
1.882 \\
\end{array}$ & $\begin{array}{r}20,42 \\
21,90 \\
23,85 \\
26,62 \\
560,50 \\
18,95 \\
14,40 \\
12,17 \\
10,90 \\
13,92 \\
11,42 \\
3,65\end{array}$ \\
\hline Total Barrios del Norte & 62.685 & 741,70 \\
\hline $\begin{array}{l}\text { Sector Sur } \\
\text { Fray Albino } \\
\text { Miraflores } \\
\text { Polígono del Guadalquivir }\left({ }^{*}\right)\end{array}$ & $\begin{array}{r}25.562 \\
10.778 \\
1.344 \\
\end{array}$ & $\begin{array}{l}75,22 \\
55,40 \\
10,30 \\
-\end{array}$ \\
\hline 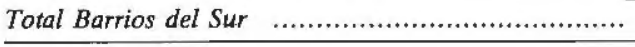 & 37.784 & 140,92 \\
\hline $\begin{array}{l}\text { Fuensanta } \\
\text { Levante Norte } \\
\text { Fátima } \\
\text { Viñuela } \\
\text { Cairo } \\
\text { Cañero } \\
\text { Santuana } \\
\text { Benito de Baños } \\
\text { Arcángel }\end{array}$ & $\begin{array}{r}16.278 \\
13.962 \\
13.903 \\
10.386 \\
7.532 \\
6.725 \\
6.575 \\
6.038 \\
4.206\end{array}$ & $\begin{array}{r}50,72 \\
48,52 \\
50,62 \\
14,35 \\
16,50 \\
43,02 \\
27,12 \\
12,30 \\
8,45\end{array}$ \\
\hline 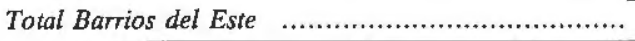 & 85.605 & 271,60 \\
\hline $\begin{array}{l}\text { Ciudad Jardín } \\
\text { Parque Figueroa } \\
\text { Parque Cruz Conde } \\
\text { Electromecánicas } \\
\text { Palmeras } \\
\text { Vallellano } \\
\text { Huerta de la Marquesa } \\
\text { Vista Alegre } \\
\text { Polígono de Poniente } \\
\text { Olivos Borrachos }\end{array}$ & $\begin{array}{r}23.568 \\
6.772 \\
6.358 \\
4.528 \\
3.004 \\
2.805 \\
2.134 \\
2.059 \\
1.861 \\
945 \\
\end{array}$ & $\begin{array}{r}43,47 \\
29,92 \\
29,37 \\
17,62 \\
9,85 \\
4,45 \\
9,37 \\
16,50 \\
11,10 \\
7,10\end{array}$ \\
\hline 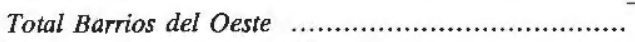 & 54.034 & 178,75 \\
\hline
\end{tabular}

(*) En 1984 aún no se había producido la consolidación definitiva de este barrio.

Fuente: Padrón Municipal de Habitantes y Elaboración propia. 
del Guadalquivir y Palmeras; barrios que tienen en común albergar a personas de condición socioeconómica extrema. Con esto se confirma una vez más la tesis de García Bellido y González Tamarit sobre la evidente segregación social de las ciudades españolas ${ }^{44}$, a la vez que coincidimos con Carter cuando afirma: «La segregación residencial es mayor para aquellos grupos ocupacionales con un status muy definido que para los que el status es ambiguo» ${ }^{45}$.

\section{CONCLUSIONES}

En la actualidad las divisiones territoriales existentes no responden, por lo general, a las necesidades crecientes de estudio y gestión en buena parte de las ciudades españolas. Por ello es preciso continuar la búsqueda de fórmulas que contribuyan a la pronta resolución del problema. La definición y delimitación del barrio puede ser una solución efectiva a corto y largo plazo ya que, entre otras cosas, esta realidad urbana goza de una sólida trayectoria histórica.

La aplicación de la metodología descrita sintéticamente demuestra la posibilidad de acceder a una división territorial de la ciudad, que sea vivida por la mayor parte de los vecinos, más racional y objetiva que las existentes hasta el momento (en su mayoría administrativas) y potencialmente utilizable por la Administración Local en la ordenación de la ciudad. En este entido, y en función de necesidades operativas, tal división ofrece la posibilidad de crear unidades mayores (que cumplirían la función de los actuales distritos) a partir de la agrupción de barrios, y no al contrario como viene siendo habitual. Asimismo, el hecho de haber respetado — - n la medida de lo posible- las secciones censales, facilita la recogida de la información básica a nivel demográfico, socioeconómico, educativo, etc. a partir de los Censos y Padrones, y la convierte, por lo tanto, en una aceptable división a la hora de elaborar estudios de diversa índole.

Por último, la aceptación del barrio como unidad geográfica diferenciada en el seno de la ciudad tiene, además, dos consecuencias muy positivas: en primer lugar se trata de un instrumento útil con vistas a una descentralización administrativa -especialmente en las grandes ciudades-; en segundo término, facilitará al vecindario su participación en la gestión y el conocimiento de la realidad urbana. Para que estos logros sean efectivos no bastará con la mera aceptación de la unidad barrio, sino que habrá que dotarla de una definición jurídica y de un uso técnico continuado por parte de los expertos.

44. García Bellido, J. y González Tamarit, L.: Para comprender la ciudad. Madrid, Nuestra Cultura, 1980 ( $2 .^{\mathrm{a}}$ ed. $)$, pp. 63 y ss.

45. Carter, H.: El estudio de la Geografía Urbana. Madrid, Instituto de Estudios de Administración Local, 1974, p. 291. 